\title{
A CHARACTERIZATION OF THE CONNECTIVITY OF A MANIFOLD IN TERMS OF LARGE OPEN CELLS
}

\author{
R. RICHARD SUMMERHILL
}

ABSTRACT. If $k$ and $n$ are integers, $0 \leq k \leq n-3$, and $M^{n}$ is a topological $n$-manifold without boundary, it is shown that $M$ is $k$-connected if and only if there is a "tame" $(n-k-1)$-dimensional closed subset $X$ in $M$ such that $M-X$ is homeomorphic to $E^{n}$.

1. Introduction. In [1] Morton Brown proved that any compact connected topological $n$-manifold $M^{n}$ without boundary is the continuous image of an $n$-cell such that the boundary of the cell is taken onto a subset of $M$ having dimension $\leq n-1$ and the interior of the cell is taken homeomorphically onto the rest of $M$. Thus any compact $n$-manifold can be obtained from euclidean $n$-space $E^{n}$ by simply (and carefully) pasting on a space $X$ of dimension $\leq n-1$. We examine here the question of just how small the dimension of $X$ can be made and show that this question is directly related to the connectivity of $M$.

By a (topological) $n$-manifold $M^{n}$, we mean a separable metric space, each point of which has an open neighborhood homeomorphic to $E^{n}$ (we shall assume throughout that all manifolds are without boundary). A euclidean chart for $M$ is a pair $(h, W)$ where $W$ is an open subset of $M$ and $h: E^{n} \rightarrow W$ is a homeomorphism. For any chart $(h, W)$ of $M$ and any real number $t>0$, let $W_{t}=h\left(C_{t}\right)$ where $C_{t}$ is the (closed) $n$-cell in $E^{n}$ with center 0 and radius $t$.

A closed subset $X$ of a topological space $Y$ is a $Z_{1}$-set if for every nonempty 1-connected open subset $U$ of $Y, U-X$ is nonempty and 1-connected. If $Y$ is a metric space, $X$ is a subset of $Y$, and $\epsilon>0$, let $N(X, \epsilon)$ denote the set of points in $Y$ whose distance from $X$ is less than $\epsilon$. An $\epsilon$-push of the pair $(Y, X)$ is a homeomorphism $h$ of $Y$ for which an $\epsilon$-isotopy $H$ of $Y$ exists satisfying: $H_{0}=1, H_{1}=h$, and $H_{t} \mid Y-N(X, \epsilon)=1$ for each

Received by the editors October 27, 1972 and, in revised form, December 29, 1972.

AMS (MOS) subject classifications (1970). Primary 57 A99, 57 A15; Secondary $57 \mathrm{~A} 30,57 \mathrm{~A} 65$. 
$t$ in $[0,1]$. A closed subset $X$ of $E^{n}$ is said to be a strong $Z_{m}$-set in $E^{n}$ ( $m$ an integer, $-1 \leq m<n$ ) if for each compact subpolyhedron $Q$ of $E^{n}$ having dimension $\leq m+1$, and each $\epsilon>0$, there exists an $\epsilon$-push $h$ of $\left(E^{n}\right.$, $X \cap Q)$ such that $h(X) \cap Q=\varnothing$. Heuristically, one should think of a strong $Z_{m}$-set in $E^{n}$ as a "tame" subset of $E^{n}$ having dimension $\leq n-m-2$ : the strong $Z_{m}$-sets in $E^{n}, m \geq 1$, are precisely those which are $Z_{1}$-sets and have dimension $\leq n-m-2$ (see 2.1 below). A closed subset $X$ of an $n$-manifold $M$ is a strong $Z_{m}$-set in $M$ if for each point $x$ in $X$ there is a euclidean chart $(h, W)$ for $M$ such that $x \in W$ and $h^{-1}(X)$ is a strong $Z_{m}$-set in $E^{n}$.

The main result of this paper is

Theorem 1.1. Let $k$ and $n$ be integers, $0 \leq k \leq n-3$, and let $M^{n}$ be an $n$-manifold. Then $M$ is $k$-connected if and only if there is a strong $Z_{k-1}$-set $X$ in $M$ such that $M-X$ is homeomorphic to $E^{n}$.

Our proof in the "if" direction is basically a general position argument: A singular $k$-sphere in $M$ is pushed off $X$, and hence into $M-X$, where it bounds. The proof in the "only if" direction is similar to Morton Brown's proof in [1]. We start with an open cell in $M$ and engulf a dense $k$-dimensional subset of $M$ whose complement is $(n-k-1)$-dimensional. The complement of the open cell (after the engulfing) is a strong $Z_{k-1}$-set in $M$. A more useful form of 1.1 is

Theorem 1.2. Let $M^{n}$ be an n-manifold.

(1) $M$ is connected if and only if there is a closed subset $X$ of $M$ such that $\operatorname{dim} X \leq n-1$ and $M-X$ is homeomorphic to $E^{n}$.

(2) If $n \geq 4$, then $M$ is simply connected if and only if there is a closed subset $X$ of $M$ such that $\operatorname{dim} X \leq n-2$ and $M-X$ is homeomorphic to $E^{n}$.

(3) If $2 \leq k \leq n-3$, then $M$ is $k$-connected if and only if there is a $Z_{1}$. set $X$ in $M$ such that $\operatorname{dim} X \leq n-k-1$ and $M-X$ is homeomorphic to $E^{n}$.

Throughout the remainder of this paper let $M^{n}$ be a fixed n-manifold with metric $d$.

In $\$ 2$ we shall discuss strong $Z_{m}$-sets in $M$ and in $\$ 3$ we present the proofs of 1.1 and 1.2 .

2. Tame sets in topological manifolds. First we give a characterization of strong $Z_{m}$-sets in terms of dimension and local homotopy properties.

Lemma 2.1. Let $m$ be an integer, $-1 \leq m<n$, and let $X$ be a closed subset of $M$.

(1) If $X$ is a strong $Z_{m}$-set in $M$, then $\operatorname{dim} X \leq n-m-2$. 
(2) If $\operatorname{dim} X \leq n-1$, then $X$ is a strong $Z_{-1}$-set in $M$.

(3) If $\operatorname{dim} X \leq n-2$ and $n \neq 3$, then $X$ is a strong $Z_{0}$-set in $M$.

(4) If $\operatorname{dim} X=n-m-2 \leq n-3, n \neq 4$, and $X$ is a $Z_{1}$-set in $M$, then $X$ is a strong $Z_{m}$-set in $M$.

Proof. For the case $M=E^{n}$, this lemma is a precise restatement of 3.1 of [5]. To prove the lemma for an arbitrary manifold, one need only look at charts and apply the case $M=E^{n}$.

Lemma 2.2. Let $X$ be a strong $Z_{m}$-set in $M$ and let $Q$ be a compact $(m+1)$-dimensional polyhedron. Then any map $f: Q \rightarrow M$ is homotopic to a map $g: Q \rightarrow M-X$.

Proof. There exist finitely many euclidean charts $\left(h_{1}, W_{1}\right), \ldots,\left(h_{r}, W_{r}\right)$ such that $f(Q) \subset \bigcup_{i=1}^{r} W_{i}$ and $h_{i}^{-1}(X)$ is a (possibly empty) strong $Z_{m}$-set in $E^{n}$. Furthermore, there exists a real number $t$ such that $f(Q) \subset W_{1 t} \cup \ldots$ $\cup W_{r t}$ where $W_{i t}=h_{i}\left(C_{t}\right)$ for each $i \leq r$. For each $i \leq r$, let $Q_{i}$ be a compact subpolyhedron of $Q$ such that $f^{-1}\left(W_{i t}\right) \subset Q_{i} \subset f^{-1}\left(W_{i}\right)$. We shall construct, by induction, a sequence of maps $f_{1}, \ldots, f_{r}$ from $Q$ into $M$ such that

(1) $f$ is homotopic to $f_{1}$, and $f_{i}$ is homotopic to $f_{i+1}$ for each $i \leq r$,

(2) $f_{i}\left(Q_{j}\right) \subset W_{j}$ for each $i, j \leq r$, and

(3) $f_{i}\left(Q_{1} \cup \ldots \cup Q_{i}\right) \cap X=\varnothing$ for each $i \leq r$.

Then $g=f_{r}$ is a map homotopic to $f$ and $g(Q) \cap X=\varnothing$. To start the induction consider the map $f \mid Q_{1}: Q_{1} \rightarrow W_{1}$. By the simplicial approximation theorem there is a map $f_{1}^{\prime}: Q \rightarrow M$ such that $f_{1}^{\prime}$ is homotopic to $f, f_{1}^{\prime}\left(Q_{j}\right) \subset W_{j}$ for each $j \leq r$, and $f_{1}^{\prime} \mid Q_{1}: Q_{1} \rightarrow W_{1}$ is PL where $W_{1}$ has the PL structure induced by $h_{1}$. Since $h_{1}^{-1}(X)$ is a strong $Z_{m}$-set in $E^{n}$, there is a homeomorphism $g_{1}: M \rightarrow M$, isotopic to the identity, such that $g_{1} f_{1}^{\prime}\left(Q_{j}\right) \subset W_{j}$ for each $j \leq r$ and $g_{1} f_{1}^{\prime}\left(Q_{1}\right) \cap X=\varnothing$. Then $f_{1}=g_{1} f_{1}^{\prime}$ is homotopic to $f, f_{1}\left(Q_{j}\right) \subset W_{j}$ for each $j \leq r$, and $f_{1}\left(Q_{1}\right) \cap X=\varnothing$. Now suppose that $f_{i-1}$ has been chosen, $i \leq r$, and consider the map $f_{i-1} \mid Q_{i}: Q_{i} \rightarrow W_{i}$. By the simplicial approximation theorem there is a map $f_{i}^{\prime}: Q \rightarrow M$ such that $f_{i}^{\prime}$ is homotopic to $f_{i-1}$, $f_{i}^{\prime}\left(Q_{j}\right) \subset W_{j}$ for each $j \leq r, f_{i}^{\prime}\left(Q_{1} \cup \cdots \cup Q_{i-1}\right) \cap X=\varnothing$, and $f_{i}^{\prime} \mid Q_{i}: Q_{i} \rightarrow W_{i}$ is PL where $W_{i}$ has the structure induced by $h_{i}$. Since $h_{i}^{-1}(X)$ is a strong $Z_{m}$-set in $E^{n}$, there is a homeomorphism $g_{i}: M \rightarrow M$, isotopic to the identity, such that $g_{i} f_{i}^{\prime}\left(Q_{j}\right) \subset W_{j}$ for each $j \leq r, g_{i}$ is fixed on $f_{i}^{\prime}\left(Q_{1} \cup \cdots \cup Q_{i-1}\right)$, and $g_{i} f_{i}^{\prime}\left(Q_{i}\right) \cap X=\varnothing$. Then $f_{i}=g_{i} f_{i}^{\prime}$ is homotopic to $f, f_{i}\left(Q_{j}\right) \subset W_{j}$ for each $j \leq r$, and $f_{i}\left(Q_{1} \cup \cdots \cup Q_{i}\right) \cap X=\varnothing$.

We now construct a $k$-dimensional dense subset of $E^{n}$. Let $k$ be an integer, $0 \leq k<n$, and let $J_{0}$ be a rectilinear PL triangulation of $E^{n}$ such that all the $n$-simplexes of $J_{0}$ have the same diameter. For each integer 
$i \geq 1$, let $J_{i}$ be the $i$ th barycentric subdivision of $J_{0}$ and let $J_{i}^{k}$ be the $k$-skeleton of $J_{i}$. Finally, set $\widetilde{B}_{n}^{k}=\bigcup_{i=1}^{\infty}\left|J_{i}^{k}\right|$ and $\widetilde{P}_{n}^{n-k-1}=E^{n}-\widetilde{B}_{n}^{k}$. Clearly $\widetilde{B}_{n}^{k}$ is $k$-dimensional and $\tilde{P}_{n}^{n-k-1}$ is $(n-k-1)$-dimensional. Moreover, $\widetilde{B}_{n}^{k}$ satisfies a very nice "absorption" property:

Lemma 2.3. Let $Q$ be a compact k-dimensional subpolyhedron of $E^{n}$, let $U$ be an open subset of $E^{n}$, and let $\epsilon>0$. Then there is a homeomorphism $h: E^{n} \rightarrow E^{n}$, fixed outside $U$ and moving points a distance less than $\epsilon$, such that $h(Q \cap U) \subset \widetilde{B}_{n}^{k}$.

Proof. This follows directly from Lemma 4.5 of [5].

Lemma 2.4. A closed subset $X$ of $E^{n}$ which is contained in $\tilde{P}_{n}^{n-k-1}$ is a strong $Z_{k-1}$ set in $E^{n}$.

Proof. Let $Q^{k}$ be a compact $k$-dimensional subpolyhedron of $E^{n}$, let $U$ be an open set in $E^{n}$ containing $X \cap Q$, and let $\epsilon>0$. By 2.3, there is a homeomorphism $h$, fixed outside $U$ and moving points a distance less than $\epsilon$, such that $h(Q \cap U) \subset \widetilde{B}_{n}^{k}$. In particular, $h^{-1}(X) \cap Q=\varnothing$. Hence if $h^{-1}$ were $\epsilon$-isotopic to the identity by an isotopy fixing $E^{n}-U$, then $X$ would be a strong $Z_{k-1}$-set. But the existence of such an isotopy follows easily from the results in [3].

We now construct a dense $k$-dimensional subset of $M$. Let $\left\{\left(h_{i}, W_{i}\right)\right\}$ be a countable collection of euclidean charts such that $M=\bigcup_{i=1}^{\infty} W_{i}$ and let $\widetilde{B}_{M}^{k}=\bigcup_{i=1}^{\infty} h_{i}\left(\widetilde{B}_{n}^{k}\right)$ and $\widetilde{P}_{M}^{n-k-1}=M-\widetilde{B}_{M}^{k}$. Clearly $\widetilde{B}_{M}^{k}$ is $k$-dimensional and $\tilde{P}_{M}^{k}$ is $(n-k-1)$-dimensional. Moreover, $\widetilde{B}_{M}^{k}$ can be written as the countable union of compact subsets $\left\{\tilde{B}_{(i)}^{k}\right\}_{i=1}^{\infty}$ of $M$ having the following property: if $i \geq 1$, then there exists $j \geq 1$ such that $\tilde{B}_{(i)}^{k} \subset W$, and $h_{j}^{-1}\left(\tilde{B}_{(i)}^{k}\right)$ is a compact subpolyhedron of $E^{n}$ having dimension $\leq k$. For the remainder of this paper we fix $\left\{\left(h_{i}, W_{i}\right)\right\}, \widetilde{B}_{M}^{k}, \tilde{P}_{M}^{n-k-1}$, and $\left\{\tilde{B}_{(i)}^{k}\right\}$ as above.

The final lemma of this section follows directly from 2.4.

Lemma 2.5. A closed subset of $M$ which is contained in $\widetilde{P}_{M}^{n-k-1}$ is a strong $Z_{k-1}$-set in $M$.

3. Connectivity in topological manifolds. Let $X$ be a $Z_{k-1}$-set in $M$ where $0 \leq k<n$ and let $f: S^{k} \rightarrow M$ be a map of the $k$-sphere into $M$. By Lemma 2.2, $f$ is homotopic to a map $g: S^{k} \rightarrow M-X$. If $M-X$ is homeomorphic to $E^{n}$, then $g$ extends to a map of the $(k+1)$-ball into $M-X$ and hence $f$ is null-homotopic in $M$. This proves

Theorem 3.1. If there is a strong $Z_{k-1}$ set $X$ in $M^{n}, 0 \leq k<n$, such that $M-X$ is homeomorphic to $E^{n}$, then $M$ is $k$-connected. 
To prove the converse of 3.1 (for codimension $\geq 3$ ), we require the following engulfing lemma; its proof is almost precisely the same as the proof of Lemma 1 of [2] and therefore we leave the details to the reader.

Lemma 3.2. Let $k$ be an integer, $0 \leq k \leq n-3$, and let $M^{n}$ be $k$-connected. Let $Q$ be a compact subset of $M$ such that for some chart $(g, U)$ of $M, Q \subset U$ and $g^{-1}(Q)$ is a k-dimensional subpolyhedron of $E^{n}$. If $(h, W)$ is a euclidean chart for $M$ and $t$ is a positive real number, then there is $a$ homeomorphism $f$ of $M$ such that $f \mid W_{t}=1$ and $f(W) \supset Q$.

Lemma 3.3. If $k$ is an integer, $0 \leq k \leq n-3$, and $M^{n}$ is $k$-connected, then there is a euclidean chart $(g, U)$ of $\bar{M}$ such that $U$ contains $\widetilde{B}_{M}^{k}$.

Proof. Consider the compact subsets $\widetilde{B}_{(i)}^{k}$ of $M$ as defined in the previous section and let $(h, W)$ be any euclidean chart of $M$. By Lemma 3.2, there is a homeomorphism $f_{1}$ of $M$ such that $f_{1}(W) \supset \widetilde{B}_{(1)}^{k}$. Since $\widetilde{B}_{1}^{k}$ is compact, there is a real number $t_{1} \geq 1$ such that $f_{1}\left(W_{t_{1}}\right) \supset \widetilde{B}_{(1)}^{k}$. Applying the lemma again, there is a homeomorphism $f_{2}$ of $M$ such that $f_{2} \mid f_{1}\left(W_{t_{1}}\right)=1$ and $f_{2} f_{1}(W) \supset \widetilde{B}_{(2)}^{k}$. Let $t_{2} \geq \max \left\{t_{1}, 2\right\}$ and such that $f_{2} f_{1}\left(W_{t_{2}}\right) \supset \widetilde{B}_{(2)}^{k}$. Continuing inductively, there is a sequence $\left\{f_{i}\right\}$ of homeomorphisms of $M$ and a sequence $t_{1} \leq t_{2} \leq \cdots$ of real numbers such that

(1) $f_{i} \mid f_{i-1} \cdots f_{1}\left(W_{t_{i-1}}\right)=1$,

(2) $f_{i} \cdots f_{1}\left(W_{t_{i}}\right) \supset \widetilde{B}_{(i)}^{k}$, and

(3) $t_{i} \geq i$

for each $i \geq 1$. Then $f=\lim _{i \rightarrow \infty} f_{i} \cdots f_{1} \mid W$ is an embedding of $W$ into $M$ such that $f(W) \supset \widetilde{B}_{M}^{k}$ and hence $(g, U)=(f h, f(W))$ is a euclidean chart satisfying the desired condition.

Theorem 3.4. If $k$ is an integer, $0 \leq k \leq n-3$, and $M^{n}$ is $k$-connected, then there is a strong $Z_{k-1}$-set $X$ in $M$ such that $M-X$ is homeomorphic to $E^{n}$.

Proof. Let $(g, U)$ be a chart in $M$ with $\widetilde{B}_{M}^{k} \subset U$. Then $X=M-U$ is closed in $M$ and is contained in $\widetilde{P}_{M}^{n-k-1}$. By $2.5, X$ is a strong $Z_{k-1}$-set in $M$.

Proof of Theorem 1.1. Apply 3.1 and 3.4.

Proof of Theorem 1.2. (1) If $M$ is connected, the result follows from the proof of the Theorem in [1]. The proof given there is an elementary form of engulfing and does not require codimension three. The converse is trivial since 0 -spheres can easily be pushed off codimension 1 subsets. The proofs of (2) and (3) follow easily from 3.4 and 2.1.

We end the paper with a rather strange corollary to 3.3 . While not direct- 
ly related to the above results, it does show that the subset $\widetilde{B}_{M}^{k}$ of $M$ is independent of the charts $\left\{\left(h_{i}, W_{i}\right)\right\}$ and, in fact, depends only on the connectivity of $M$.

Corollary 3.5. Let $k$ be an integer, $0 \leq k \leq n-3, n \neq 4$, and let $M$ be k-connected. Then $\widetilde{B}_{M}^{k}$ and $\widetilde{B}_{n}^{k}$ are homeomorphic.

Proof. Let $(g, U)$ be a chart in $M$ such that $\widetilde{B}_{M}^{k} \subset U$. By 5.4 and 2.5 of [5], there is a homeomorphism of $E^{n}$ which takes $g^{-1}\left(\widetilde{B}_{M}^{k}\right)$ onto $\widetilde{B}_{n}^{k}$.

\section{REFERENCES}

1. M. Brown, A mapping theorem for untriangulated manifolds, Topology of 3Manifolds and Related Topics (Proc. Univ. of Georgia Inst., 1961), Prentice-Hall, Englewood Cliffs, N. J., 1962, pp. 92-94. MR 28 \#1599.

2. E. H. Connell, A topological H-cobordism theorem for $n \geq 5$, Illinois J. Math. 11 (1967), 300-309. MR 35 \#3673.

3. R. D. Edwards and R. Kirby, Deformations of spaces of imbeddings, Ann. of Math. (2) 93 (1971), 63-88.

4. R. Geoghegan and R. R. Summerhill, Concerning the shapes of finite-dimensional compacta, Trans. Amer. Math. Soc. 179 (1973), 281-292.

5. - Pseudo-boundaries and pseudo-interiors in euclidean spaces and topological manifolds, Trans. Amer. Math. Soc. 194 (1974), 141-165.

6. - Infinite-dimensional methods in finite-dimensional geometric topology, Bull. Amer. Math. Soc. 78 (1972), 1009-1014.

7. M. H. A. Newman, The engulfing theorem for topological manifolds, Ann. of Math. 84 (1966), 555-571.

8. R. R. Summerhill, General position for closed subsets of Euclidean space General Topology and Appl. 3 (1973), 339-345.

DEPARTMENT OF MATHEMATICS, KANSAS STATE UNIVERSITY, MANHATTAN, KANSAS 66506 